\title{
Fluconazole reduced the rate of recurrence of vulvovaginal candidiasis
}

Sobel JD, Wiesenfeld HC, Martens M, et al. Maintenance fluconazole therapy for recurrent vulvovaginal candidiasis. N Engl J Med 2004;351:876-83.

\section{What is the efficacy of fluconazole for recurrent vulvovaginal candidiasis (VC)?}

\section{METHODS}

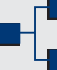

Design: randomised placebo controlled trial.

Q2.

Allocation: unclear.

Blinding: blinded (patients and healthcare providers).

Follow up period: 12 months.

Setting: multiple centres in the US

Patients: 387 women $\geqslant 18$ years of age (mean age $34 y, 67 \%$ white) who had active, acute candida vaginitis (severity score

$\geqslant 3$ ), a positive result on microscopic examination of vaginal secretions, $\geqslant 4$ episodes of candida vaginitis in the previous 12 months, and were clinically cured (severity score $<3$ ) at 14 days after receiving 3 sequential doses of fluconazole, $150 \mathrm{mg}$, at 72 hour intervals. Exclusion criteria: lack of confirmation of microscopic findings by culture, pregnancy, mixed infections or known seropositivity for HIV, or use of antifungal agents in the previous 4 weeks.

R Intervention: a single oral dose of fluconazole, $150 \mathrm{mg}$

1. $(n=170)$, ${ }^{*}$ or an oral placebo tablet taken weekly for 6 months $(n=173){ }^{*}$

12 Outcomes: clinical recurrence of VC (severity score $\geqslant 3$ plus vaginal cultures positive for yeast) at the end of the 6 month treatment period and at 12 months, and time to clinical and mycologic recurrence of $\mathrm{VC}$.

IIU Patient follow up: 373 of 387 women (96\%) were included in the modified intention to treat (ITT) analysis. Efficacy analyses were based on data from 283 women $(73 \%)$ for clinical recurrence at 6 months and 263 women (68\%) for clinical recurrence at 1 year.

*Data on allocation status only available for 343 women included in the efficacy analysis.

\section{MAIN RESULTS}

Efficacy analyses showed that women in the fluconazole group had lower rates of clinical recurrence of VC at 6 months $\{9 \% \vee 64 \%$,

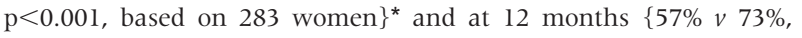

For correspondence: Dr J D Sobel, Division of Infectious Diseases, Wayne State University School of Medicine, Detroit, MI, USA. jsobel@med. wayne.edu

Source of funding: Pfizer Pharmaceutical. $\mathrm{p}<0.001$, based on 263 women $^{*}$ than those in the placebo group. The authors reported similar findings for the modified ITT analysis (no data were reported in article). Median time to clinical recurrence was 10.2 months in the fluconazole group and 4.0 months in the placebo group $(\mathrm{p}<0.001$; Kaplan Meier analysis). Median time to mycologic recurrence was 8.4 months in the fluconazole group and 1.9 months in the placebo group ( $\mathrm{p}<0.001$; Kaplan Meier analysis).

\section{CONCLUSION}

In women with recurrent vulvovaginal candidiasis (VC), fluconazole reduced the rate of recurrence of VC and increased time to clinical and myocologic recurrence.

${ }^{*}$ Calculated from data in original article.

\section{Commentary}

M ore than half of women in the US will experience $\geqslant 1$ episode of VC by the time they reach the age of 25 years, and about $5 \%$ of these women (nearly 1.3 million) will have recurrences throughout adulthood. ' Sobel et al investigated how to reduce recurrences in these women. To diagnose VC, women had to have symptom severity scores $\geqslant 3$ ( $0=$ absent or normal; $3=$ severe) for 3 symptoms (pruritus, irritation, and burning) and 3 signs (erythema, oedema, and excoriation or fissures) as well as a positive fungal culture. Hence, a spectrum of women with mild to severe VC symptoms and signs may have been enrolled in the study. The authors refer to "clinical cure" of VC, which is perhaps more accurately referred to as "effective suppression" of symptoms and signs. It is unclear which specific symptoms and signs were present in women categorised as having "effective suppression" or "recurrence." Also, clinicians should note that other variables commonly believed to predict VC, such as history of antibiotic and oral contraceptive use, were not associated with clinical response.

Even with this low threshold for clinical diagnosis of VC, weekly fluconazole resulted in recurrence rates of $4 \%$ ( 6 of 166 women) at 1 month and $9 \%$ ( 13 of 141 women) at 6 months. The 1 month posttreatment recurrence rate is far better than previous reports of $20 \%$ for both the usual 1 time oral dose and intravaginal medication. ${ }^{2}$ Better yet, this low recurrence rate was accompanied by a reassuring side effect and safety profile that persisted throughout the 12 month study. Overall, the clinical outcomes of this study are impressive and translate into a safe and convenient treatment for women with recurrent VC. Ease of access to fluconazole and cost were not addressed in this study. However, fluconazole is now available over the counter in the UK. In the US, where it remains a prescription drug, four $150 \mathrm{mg}$ tablets can be purchased for about US $\$ 25$ (www.drugstore.com, accessed 3 February 2005).

Gene Harkless, RN, DNSc, ANRP University of New Hampshire Durham, New Hampshire, USA

1 Ringdahl EN. Treatment of recurrent vulvovaginal candidiasis. Am Fam Physician 2000;61:3306-12, 3317.

2 Watson MC, Grimshaw JM, Bond CM, et al. Oral versus intra-vaginal imidazole and triazole anti-fungal treatment of uncomplicated vulvovaginal candidiasis (thrush). Cochrane Database Syst Rev 2001 ; (1):CD002845. 